\title{
TGR5 protects against cholestatic liver disease via suppressing the NF-кB pathway and activating the Nrf2/HO-1 pathway
}

\author{
Haojun Yang ${ }^{1 *}$, Fengyong Luo ${ }^{2 \#}$, Yi Wei ${ }^{1}$, Yuwen Jiao ${ }^{1}, J^{\prime}$ Qian ${ }^{1}$, Shuai Chen ${ }^{1}$, Yu Gong ${ }^{1}$, Liming Tang $^{1}$ \\ ${ }^{1}$ Gastrointestinal Surgery Department, The Affiliated Changzhou No. 2 People's Hospital of Nanjing Medical University, Changzhou, China; \\ ${ }^{2}$ School of Graduate, Dalian Medical University, Dalian, China \\ Contributions: (I) Conception and design: L Tang; (II) Administrative support: H Yang; (III) Provision of study materials or patients: F Luo; (IV) \\ Collection and assembly of data: S Chen, Y Wei; (V) Data analysis and interpretation: Y Jiao, Y Gong, J Qian; (VI) Manuscript writing: All authors; \\ (VII) Final approval of manuscript: All authors. \\ "These authors contributed equally to this work. \\ Correspondence to: Dr. Liming Tang. Gastrointestinal Surgery Department, The Affiliated Changzhou No. 2 People's Hospital of Nanjing Medical \\ University, No. 68 Gehu Middle Road, Changzhou 213164, China. Email: drtangliming@163.com.
}

Background: Characterized by the presence of inflammation, fibrosis, and bile duct proliferation, cholestatic liver disease (CLD) affects people of all age groups. Takeda G-protein-coupled receptor (TGR5) has been implicated in the suppression of inflammation via toll-like receptor 4 (TLR4) and nuclear factor kappa B (NF-kB). Kupffer cells and their M1 polarization play important roles in inflammation and cholestatic liver injury via production of pro-inflammatory cytokines. Nevertheless, the function of TGR5 signaling in CLD is largely unknown.

Methods: We conducted liver tissue experiments, animal experiments, serum marker testing, liver histology analysis, Kupffer cell experiments, RNA extraction and Real-time PCR, western blotting, evaluation of ROS production by flow cytometry and statistical differences were analyzed by student $t$-test using GraphPad Prism.

Results: We found that serum bile acid (BA) and TGR5 levels were elevated in patients with cholestasis cirrhosis. Knockout of TGR5 in animals significantly increased bile duct ligation (BDL)-caused liver injury through increasing oxidative stress, promoting M1-predominant polarization of Kupffer cells, and elevating the serum levels of inflammatory cytokines. In contrast, TGR5 activation inhibited ROS production, secretion of pro-inflammatory cytokines, and M1-predominant polarization of Kupffer cells. Moreover, results showed that TGR5 exerted its effects via suppressing NF- $\mathrm{B}$ signaling and activating nuclear factor 2 (Nrf2)/HO-1 signaling. Finally, the effect of TGR5 on cholestatic liver damage was also confirmed in vivo.

Conclusions: TGR5 activation protected against BDL-induced CLD by both suppressing inflammation via inhibiting the NF- $\kappa$ B pathway and reducing ROS production via activation of $\mathrm{Nrf} 2 / \mathrm{HO}-1$ signaling. These findings show the importance of TGR5 in CLD and provide new insight into therapeutic strategies for CLD.

Keywords: Cholestatic liver disease (CLD); inflammation; inflammatory cytokines; reactive oxygen species; TGR5

Submitted May 12, 2021. Accepted for publication Jun 28, 2021.

doi: $10.21037 /$ atm-21-2631

View this article at: https://dx.doi.org/10.21037/atm-21-2631 


\section{Introduction}

Cholestasis is caused by diminished bile formation, impaired bile secretion, or obstruction of bile flow (1). The pathological features of cholestasis include the presence of liver cell inflammation, necrosis, fibrosis, and bile duct proliferation (2). Liver diseases related to cholestasis are classified as cholestatic liver diseases (CLDs) (3), which may lead to cirrhosis (3) and place a significant health burden (4).

Bile duct ligation (BDL) is widely used in the study of cholestasis $(5,6)$, and bile acids (BAs) were significantly elevated in patients with cholestasis or liver cirrhosis $(6,7)$. Cholestatic pruritus is a frequent symptom in patients with CLDs, and BAs have been proposed as a putative pruritogen (8). TGR5 is the founder member of a BA receptor subclass which can be activated by several BAs (9). The M1 polarization of Kupffer cells, which are liver resident macrophages, contributes to inflammation and cholestatic liver injury through cytokines $(10,11)$, and studies have indicated that activation of nuclear factor kappa $\mathrm{B}(\mathrm{NF}-\kappa \mathrm{B})$ promotes $\mathrm{M} 1$ polarization of macrophages $(12,13) . T G R 5$ has been shown to protect against cholestasis by suppression of cytokine production (14), and activation of TGR5 leads to the inhibition of inflammatory response through suppressing Toll-like receptor 4 (TLR4) and subsequent pro-inflammatory cytokine production $(15,16)$. Further, TGR5 activation inhibited NF $-\kappa B$ and its target genes to suppress inflammation (17). These studies suggest TGR5 plays an important role in suppressing inflammation, which is one of the features of cholestasis. Previous study has demonstrated that oxidative stress occurs in the livers of humans with cholestasis (18) and that elevated BAs cause reactive oxygen species (ROS) production $(19,20)$. In addition, Deng et al. showed that TGR5 alleviated ROS partially via Nrf2/HO-1 signaling (21). These results suggest BAs/TGR5 may affect cholestasis, although there exact role in it and CLDs is still unclear and remains to be elucidated.

In the present study, using TGR5-/- animals and a specific TGR5 agonist, we identified TGR5 as a negative regulator of BDL-mediated CLD via suppressing NF- $\mathrm{KB}$ mediated inflammation, and alleviating BAs induced ROS in the liver.

We present the following article in accordance with the ARRIVE reporting checklist (available at https://dx.doi. org/10.21037/atm-21-2631).

\section{Methods}

\section{Chemicals}

INT-777 was purchased from MedChemExpress LLC (South Brunswick, NJ, Catalog number: HY-15677) and lipopolysaccharide (LPS) was purchased from Sigma (St. Louis, MO, Catalog number: L8274).

\section{Human liver tissue}

The study was approved by the Institutional Ethical Review Committee of the Affiliated Changzhou No. 2 People's Hospital of Nanjing Medical University \{No. [2020]KY125-01\}. Thirty patients with cholestatic liver cirrhosis and 30 controls were included, and liver tissue was collected immediately after resection and snap-frozen in liquid nitrogen. Written informed consent was obtained from all participants. All procedures performed in this study involving human participants were in accordance with the Declaration of Helsinki (as revised in 2013).

\section{Animal experiments}

TGR5-/- male mice were provided by Johan Auwerx and Kristina Schoonjans as gifts. Mice were kept in normal laboratory conditions and received ad libitum access to water and food. The experiment was approved by the Institutional Animal Care and Use Committee of The Affiliated Changzhou No. 2 People's Hospital of Nanjing Medical University, in compliance with institutional guidelines for the care and use of animals.

TGR5-/- and WT mice (8 weeks) were randomly divided into a BDL group and sham-operated group ( $n=20$ per group). BDL was performed aseptically under general anesthesia (ketamine $\mathrm{HCl}, 50 \mathrm{mg} / \mathrm{kg}$ ) and the abdomen was opened to identify and double ligate the bile duct with 5.0 silk sutures. Sham-operated mice received a similar procedure without ligation. The investigators were blinded to the group allocation during the experiment. On day 10, the mice were euthanized and liver tissues were collected.

\section{Serum marker testing}

The level of alanine aminotransferase (ALT), aspartate aminotransferase (AST), BAs, or total bilirubin (TBIL) was tested using commercial kits (Nanjing Jiancheng Bio). 
Activities of bepatic malondialdehyde (MDA) and catalase (CAT)

The activity of hepatic MDA or CAT was tested by commercial kits from Nanjing Jiancheng Bio. BCA was used to detect the protein concentration of the samples.

\section{Liver histology analysis}

Tissues were fixed, embedded, sectioned, and subjected to hematoxylin and eosin (HE) staining or Masson's trichrome staining.

\section{Kupffer cell experiments}

Kupffer cells were isolated as described previously. Cells were cultured in DMEM with 10\% FBS (Invitrogen). To determine cell purity, the cells were incubated with CD68 (1:100, Abcam, ab31630) and Alexa Fluor 488 conjugated second antibody sequentially (Beyotime).

\section{Lentivirus preparation}

Short hairpin RNA (shRNA) oligos targeting mouse Nrf2 (Table S1) were cloned into pLKO.1 vector (OriGene, Rockville, MD). The resulting plasmids, psPAX2 and pMD2.G, were co-transfected into 293T cells for lentivirus production.

\section{RNA extraction and real-time PCR}

RNA was isolated using TRIzol (Invitrogen). Two $\mu \mathrm{g}$ of RNA was used to synthesize cDNA using Primescript RT kit (TaKaRa). Ten ng of cDNA was used for qPCR with SYBR Green Master Mix (ABI) and ABI Prism 7300 system (ABI) with the following parameters: $94^{\circ} \mathrm{C} 25 \mathrm{~s}, 38$ cycles of $94{ }^{\circ} \mathrm{C} 7 \mathrm{~s}$, and $58^{\circ} \mathrm{C} 25 \mathrm{~s}$. Gene level was calculated using double-delta method. Primers are listed in Table S2.

\section{Western blotting}

Proteins were extracted using radioimmunoprecipitation buffer. The cytosolic or nuclear fraction was made by NE-PER Nuclear and Cytoplasmic Extraction Reagents (Thermo Fisher Scientific). Proteins were resolved on $8 \%$ SDS-PAGE, transferred to nitrocellulose membranes, and incubated at $4{ }^{\circ} \mathrm{C}$ overnight with primary antibodies (Table S3), followed by incubation with HRP-conjugated second antibodies. Protein band was detected with ECL reagents (Invitrogen) and analyzed with Image J.

\section{Evaluation of ROS production by flow cytometry}

Cells were harvested, washed with ice-cold PBS, and incubated with $10 \mu \mathrm{M}$ DCFH-DA (Beyotime) for 20 minutes, then analyzed by a flow cytometer (Millipore, Burlington, MA).

\section{Statistical analyses}

All data are expressed as mean \pm standard error. Statistical differences between two groups and among more than two groups were analyzed by student $t$-test and ANOVA test, respectively, using GraphPad Prism. A P value $<0.05$ was defined as statistically significant.

\section{Results}

\section{Serum BA levels and TGR5 expression levels were elevated} in patients with cholestasis cirrhosis

Both serum BA levels (Figure 1A) and the expression of TGR5 at mRNA level (Figure 1B) and protein level (Figure $1 C, D)$ were significantly up-regulated in the liver tissue of patients with cholestasis cirrhosis compared with controls.

\section{TGR5 knockout exacerbated BDL-caused liver damage}

To study TGR5 function in cholestasis cirrhosis, BDL was performed in mice. In wild type (WT) mice, BDL significantly increased serum levels of AST, ALT, total bile acid (TBA), and TBIL on day 1, 3, 7, and 10 (Figure $2 A, B, C, D)$. TGR5-/- significantly exacerbated BDLcaused elevation of AST, ALT, TBA, TBIL, and collagen deposition and, importantly, also decreased the mouse survival rate (Figure $2 E$ ). BDL also promoted collagen deposition (Figure $2 F$ ). These results suggest that TGR5 knockout significantly increased BDL-caused injury.

\section{TGR5-/- BDL mice showed increased oxidative stress and pro-inflammatory response}

To study how TGR5-/- exacerbated BDL-caused injury, oxidative stress and pro-inflammatory responses were evaluated. The results showed that BDL significantly elevated the MDA level, which was further increased by TGR5-/-. 

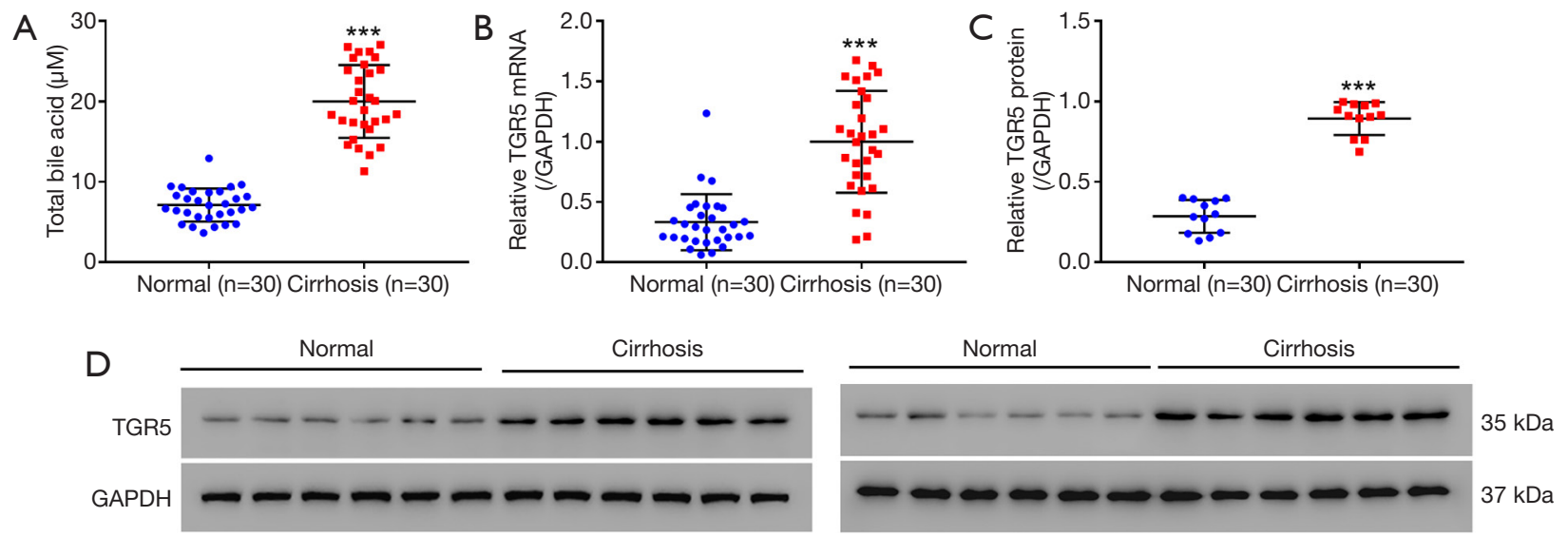

Figure 1 Patients with cholestatic cirrhosis had elevated serum bile acid and TGR5 expression levels. (A) Analysis of BAs in cholestatic cirrhosis patients $(n=30)$ and controls $(n=30)$; (B) real-time PCR analysis of TGR5 mRNA level (n=30); (C,D) Western blot analysis of TGR5 protein level $(n=12)$. The densitometric analysis is shown as $(\mathrm{D}) .{ }^{* *} \mathrm{P}<0.001$. BAs, bile acids; PCR, polymerase chain reaction.
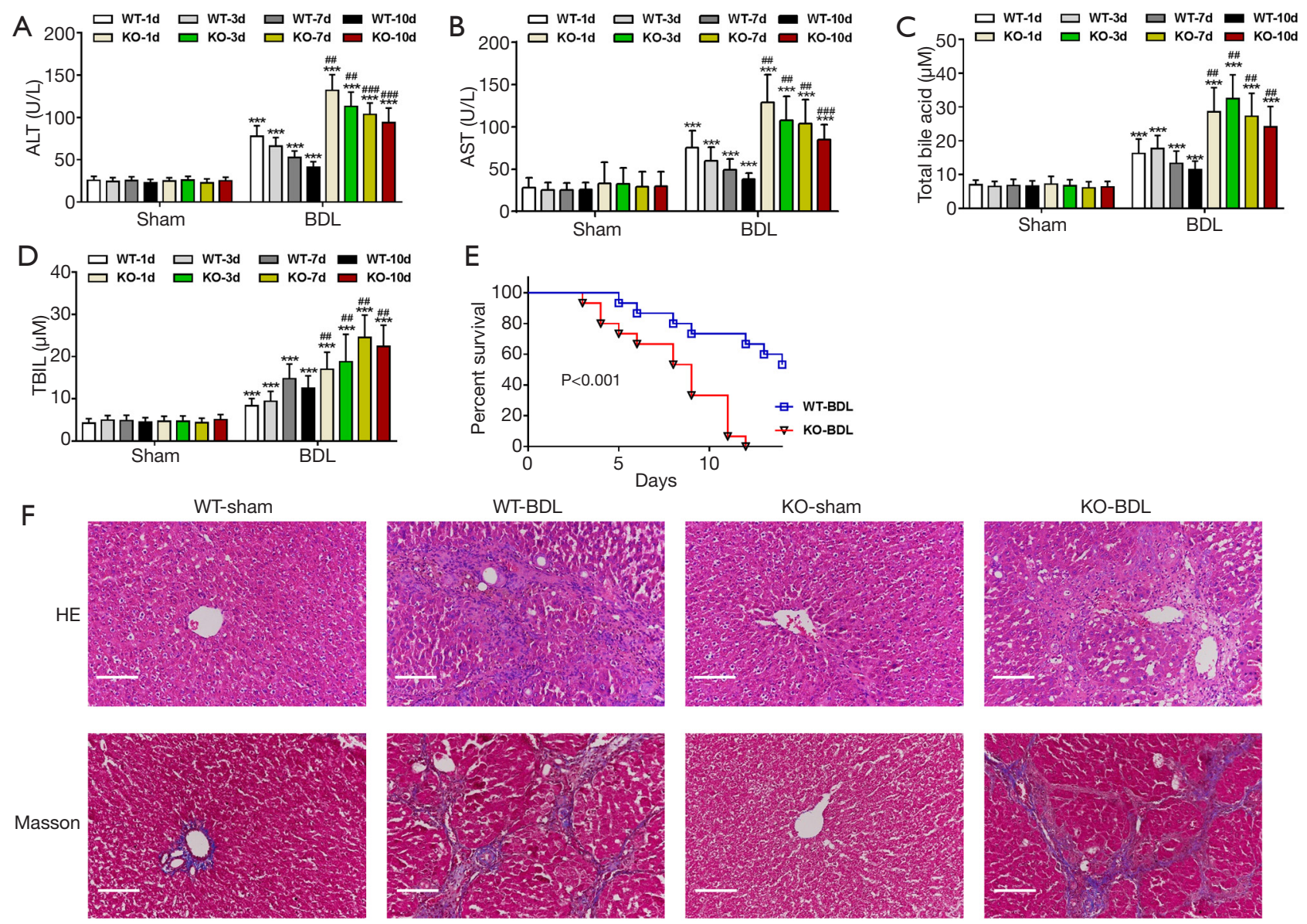

Figure 2 TGR5 knockout exacerbated BDL-induced liver injury. WT or TGR5-/- mice underwent BDL. (A,B,C,D) Levels of serum AST, ALT, total bile acid, and TBIL on day 1, 3, 7, and 10 (n=3 per group). (E) 14-day survival curve of WT in TGR5-/- BDL mice ( $\mathrm{n}=15$ per group). (F) $\mathrm{HE}$ and Masson staining of liver on day 10 (scale bar: $100 \mu \mathrm{m}$ ). ${ }^{* * *} \mathrm{P}<0.001$ (vs. sham); ${ }^{\# \#} \mathrm{P}<0.01,{ }^{\# \# \#} \mathrm{P}<0.01$ (vs. WT). BDL, bile duct ligation; ALT, alanine aminotransferase; AST, aspartate aminotransferase; TBIL, total bilirubin (TBIL); HE, hematoxylin and eosin. 

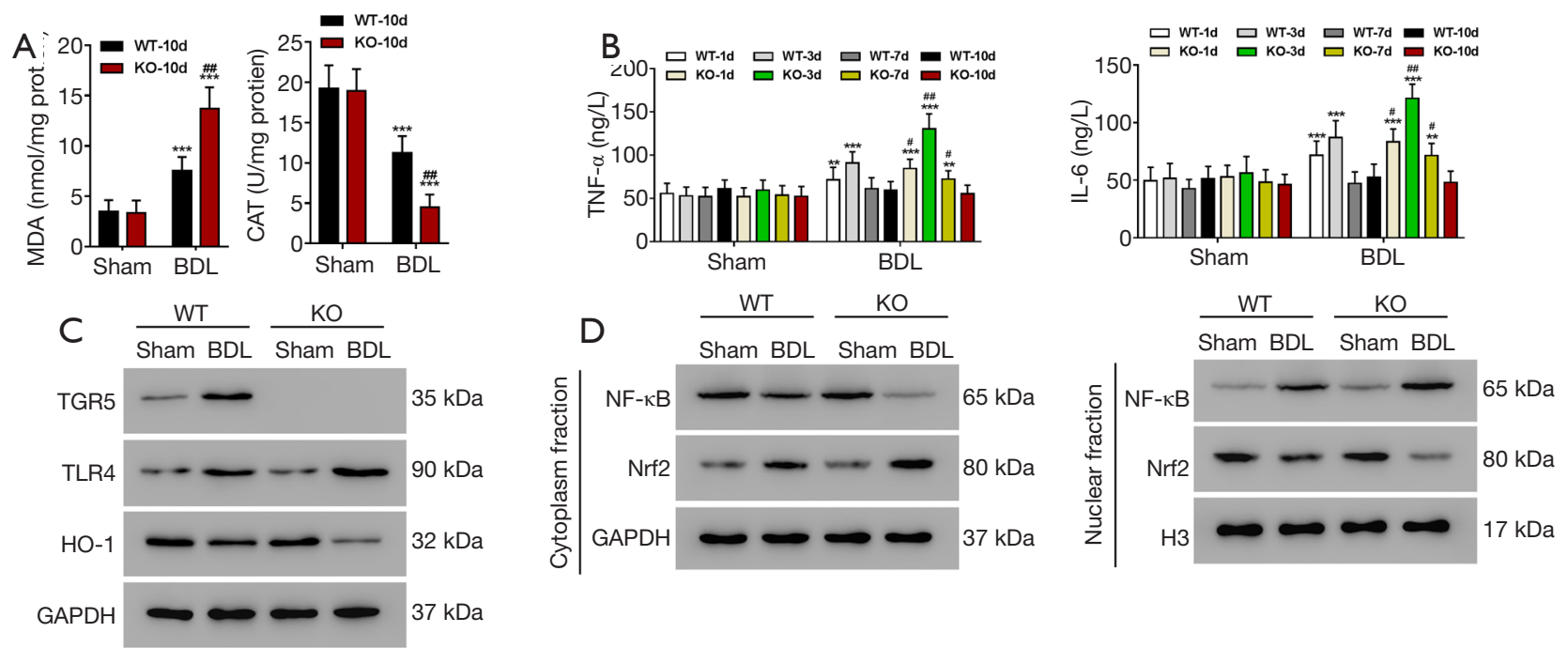

Figure 3 TGR5-/- BDL mice showed increased oxidative stress and pro-inflammatory response. WT or TGR5-/- mice underwent BDL. (A) MDA and CAT activities in liver tissues on Day 10 ( $\mathrm{n}=3$ per group); (B) TNF- $\alpha$ and IL-6 level on Day 1, 3, 7, and 10 measured by ELISA ( $\mathrm{n}=3$ per group); (C) TGR5, TLR4, and HO-1 expression in liver on Day 10 ( $\mathrm{n}=3$ per group); (D) the expression levels of NF- $\kappa \mathrm{B}$ (nuclear and cytoplasmic) and $\mathrm{Nrf2}$ (nuclear and cytoplasmic) in liver on day 10 ( $\mathrm{n}=3$ per group). ${ }^{* *} \mathrm{P}<0.01,{ }^{* * *} \mathrm{P}<0.001$ (vs. control); ${ }^{\#} \mathrm{P}<0.05,{ }^{\# \#} \mathrm{P}<0.01$ (vs. WT). BDL, bile duct ligation. MDA, malondialdehyde; CAT, catalase.

In contrast, BDL significantly decreased the level of CAT, which was further suppressed by TGR5-/- (Figure 3A). In WT mice, BDL significantly increased levels of TNF- $\alpha$ and IL-6 on day 1 and 3, which was further significantly elevated by TGR5-/- (Figure 3B). Western blotting results also showed that in WT mice, BDL dramatically enhanced TGR5 and TLR4 in liver on Day 10, but sharply suppressed HO-1. Silencing of TGR 5 further increased TLR4 but decreased HO-1 (Figure 3C), suppressed BDL-inhibited cytoplasmic NF- $\kappa \mathrm{B}$, increased BDL-induced cytoplasmic Nrf2. In contrast, silencing of TGR5 increased BDL-induced nuclear NF- $\mathrm{BB}$ but suppressed BDL-inhibited nuclear Nrf2 (Figure 3D). These findings suggest that TGR5 knockout exacerbated BDL-induced injury via promoting oxidative stress and inflammation.

\section{TGR 5 activation inbibited ROS production, secretion of pro-inflammatory cytokines, and M1-predominant polarization}

ATGR5 agonist was then used to further study the role of TGR5. Kupffer cells (Figure S1) were isolated from BDL mice, then treated with a TGR5 agonist INT-777 at a concentration of $3 \mu \mathrm{M}$ for 24 hours. Activation of TGR5 significantly decreased ROS production (Figure 4A), suppressed the expression of M1 marker iNOS2, increased the expression of M2 marker ArgI at the mRNA level (Figure 4B), and suppressed the secretion of TNF- $\alpha$ and IL-6 (Figure 4C). Activation of TGR5 remarkably inhibited the expression of TLR4, but significantly increased the expression of HO-1 (Figure 4D). Administration of INT-777 also significantly increased cytoplasmic NF$\kappa \mathrm{B}$, decreased cytoplasmic Nrf2, decreased nuclear NF$\kappa \mathrm{B}$, and increased nuclear Nrf2 (Figure 4E). These data confirm TGR5 activation suppresses ROS production and inflammation.

\section{INT-777 alleviated the effect of Nvf2 interference}

To investigate how TGR5 activation affects inflammation, Nrf2 was silenced in primary WT Kupffer cells using lentivirus (Figure S2). Nrf2-silenced WT Kupffer cells were then treated with TGR5 agonist INT-777 at a concentration of $3 \mu \mathrm{M}$ for $24 \mathrm{~h}$. The results showed that silencing of Nrf2 significantly increased ROS (Figure $5 \mathrm{~A}$ ) and M1 marker iNOS2, and decreased M2 marker ArgI at the mRNA level (Figure 5B), elevated TNF- $\alpha$ and IL-6 (Figure 5C), reduced HO-1 (Figure 5D), and increased nuclear NF-KB (Figure 5E). INT-777 alleviated the effect of Nrf2 interference. 
A
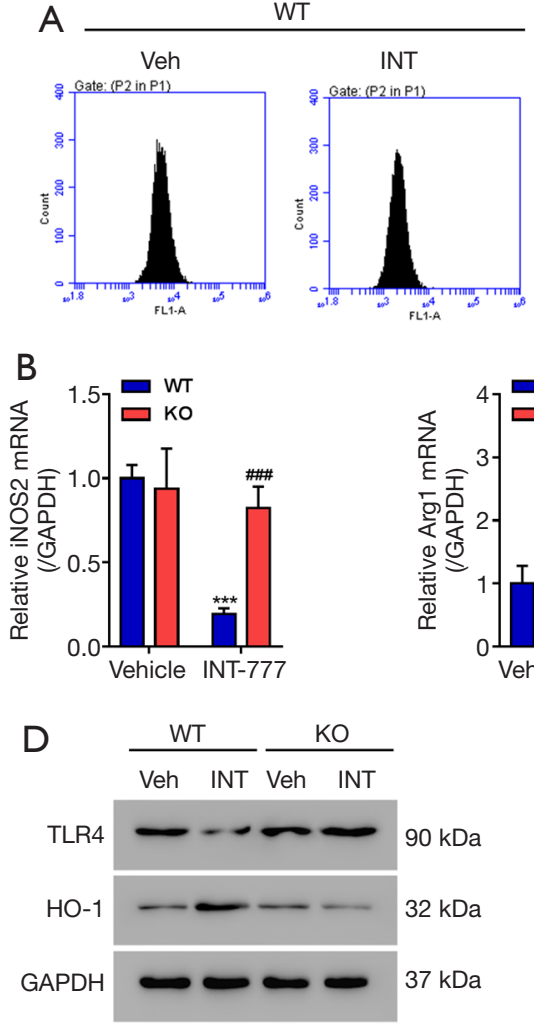

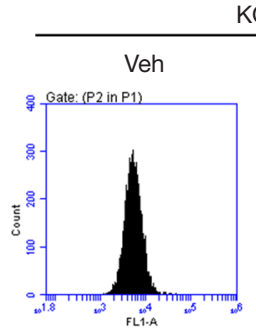

$\mathrm{KO}$

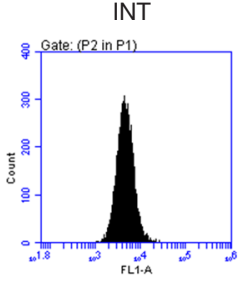

C
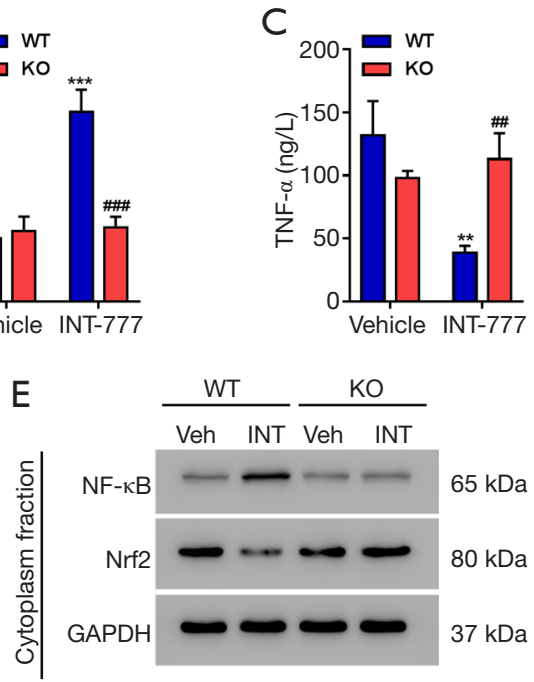
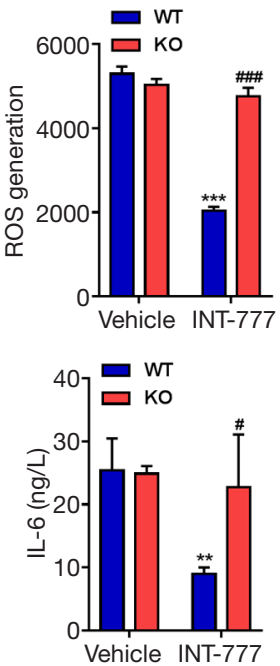

Figure 4 TGR5 agonist INT-777 inhibited ROS production, pro-inflammatory response, and M1-predominant polarization in vitro. Primary Kupffer cells from BDL mice were administered INT-777 (3 $\mu \mathrm{M})$ for 24 hours. (A) ROS levels; (B) mRNA levels of iNOS2 and Arg I; (C) TNF- $\alpha$ and IL-6 secretion; (D) protein levels of TLR4 and HO-1; (E) protein levels of NF- $\mathrm{kB}$ (nuclear and cytosol) and Nrf2 (nuclear

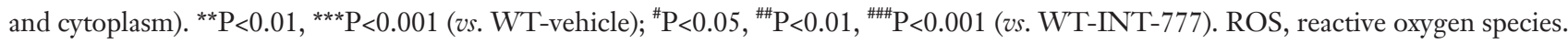

\section{Activation of TRG5 inbibited LPS-induced M1- predominant polarization, $N F-\kappa B$ nuclear translocation, and secretion of TNF- $\alpha$ and IL-6}

To further study the role of TGR5, WT mouse primary Kupffer cells were treated with LPS $(100 \mathrm{ng} / \mathrm{mL})$ and INT$777(3 \mu \mathrm{M})$ for $24 \mathrm{~h}$ because LPS has been shown to activate the TLR4/ NF- $\kappa \mathrm{B}$ signaling pathway (22). The results showed that activation of TRG5 by INT-777 significantly attenuated LPS-mediated IL- 6 and TNF- $\alpha$ increase (Figure $6 A$ ), increase the M1 marker iNOS2 and decrease of the M2 marker ArgI at the mRNA level (Figure 6B), suppression of cytoplasmic NF- $\kappa \mathrm{B}$, and increase of nuclear NF-кB (Figure 6C). These results suggest TGR5 activation abolished the effect of LPS.

\section{Discussion}

The present study demonstrated BA and TGR5 levels were elevated in patients with cholestasis cirrhosis. Using TGR5-/- mice, we further showed that knockout of TGR5 significantly increased BDL-induced liver injury via elevation of ROS, promotion of Kupffer cell M1-predominant polarization, and increase of inflammatory cytokines, which were all abolished by TGR5 activation. These findings shed light on new therapeutic strategies for CLD.

Bile acids promote the flow and excretion of bile, excess cholesterol, and waste, and these substances have been identified as "digestive surfactants" to promote the absorption of lipids (23). One common characteristic of cholestasis is elevated levels of bile acids in serum and liver. The accumulation of bile acids in the liver induces oxidative stress and mitochondrial damage, leading to the inflammatory response. The inflammatory damages the liver cells, resulting in liver fibrosis, cirrhosis and ultimately liver failure. Moreover, BAs also activate receptors to trigger downstream signaling (16). It has been shown that TGR5 was stimulated by BAs to mediate their 

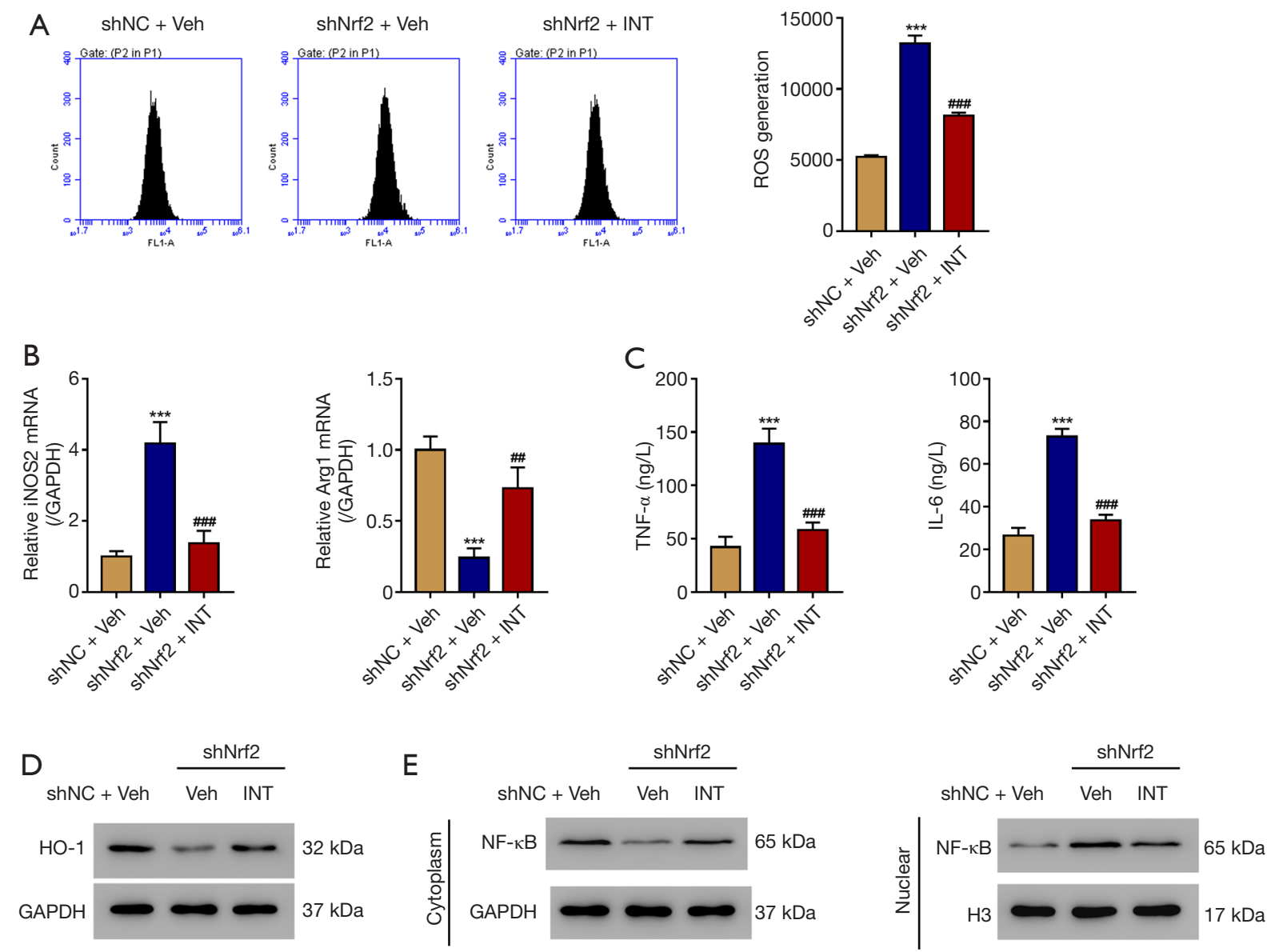

Figure $5 \mathrm{Nrf} 2$ interference alleviated the effect of INT-777. Nrf2-silencing Kupffer cells were administered with INT-777 (3 $\mu \mathrm{M}$ ) for 24 h. (A) ROS levels; (B) mRNA levels of iNOS2 and Arg I; (C) TNF- $\alpha$ and IL-6 secretion; (D) protein levels of TLR4 and HO-1; (E) protein levels of NF-kB (nuclear and cytoplasm). ${ }^{* * *} \mathrm{P}<0.001$ (vs. shNC-vehicle); ${ }^{\# \#} \mathrm{P}<0.01,{ }^{\# \# \#} \mathrm{P}<0.001$ (vs. shNrf2-vehicle). Nrf2, nuclear factor 2 ; ROS, reactive oxygen species.

effects on energy metabolism and inflammatory responses (9,24). Reich et al. reported that stimulation of TGR5 triggered ROS production, leading to bile acid-dependent cholangiocyte proliferation (25), while Keitel et al. showed that TGR5 stimulation resulted in the generation of ROS in astrocytes and neurons (26). In contrast, one study showed that activation of TGR5 by its agonist suppressed ROS production in human podocytes (27), and in the present study we found that TGR5 activation significantly suppressed ROS production. These controversial results suggest the effect of TGR5 activation on ROS production might be cell or tissue-specific.

$\mathrm{Nrf2}$ is one of the key transcription factors that mediate protection against oxidants (28). Nrf2 has been shown to promote $\mathrm{HO}-1$ promoter activity and increase $\mathrm{HO}-1$ expression (29), which plays a key role in maintaining antioxidant/oxidant homeostasis (30), and is implicated in a variety of diseases including diabetes and cancer $(31,32)$. Here we showed that TGR5 increased nuclei Nrf2, leading to HO-1 enhancement and Nrf2 knockdown attenuated TGR5 activation effect. This is consistent with the results of a previous study showing that TGR5 exerted its effects via Nrf2/HO-1 (21).

Inflammation plays an important role in various diseases including cancer, diabetes, cardiovascular disease, and CLDs (2,33-35). Previous studies showed that TLR4 plays a key role in inflammation associated with several acute and chronic diseases (36-38). All TLR signaling pathways lead to NF- $\mathrm{NB}$ activation, which controls the expression of numerous inflammatory genes (39). TLRs have been shown 
A

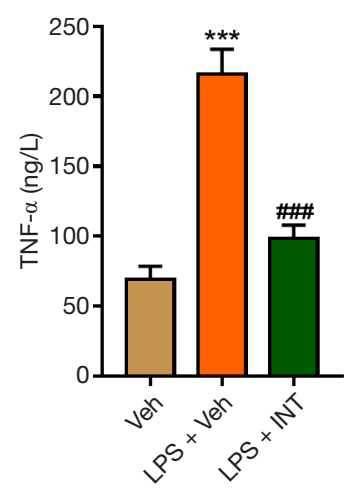

B

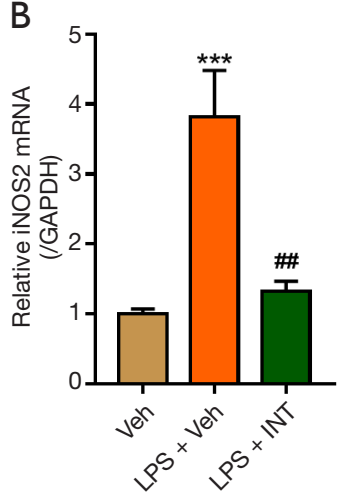

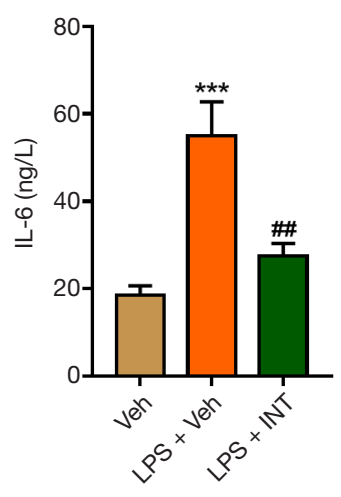

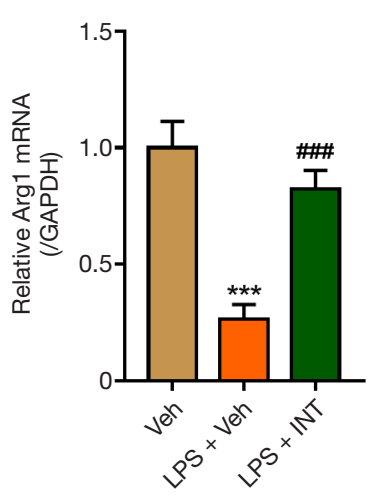

C
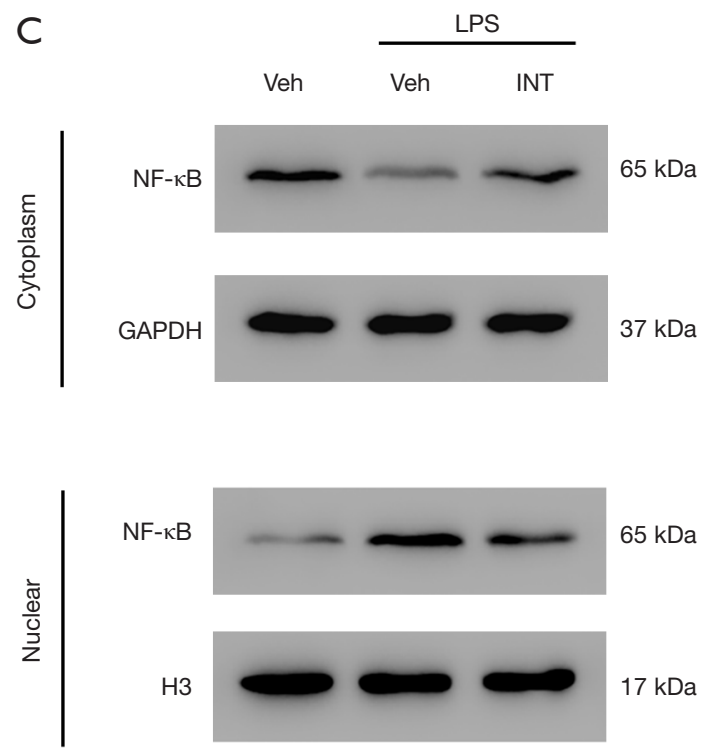

Figure 6 Activation of TGR5 by INT-777 inhibited LPS-induced M1-predominant polarization, NF-кB nuclear translocation, and secretion of inflammatory cytokines. WT primary Kupffer cells were isolated and administered LPS or INT-777. (A) TNF- $\alpha$ and IL-6 secretion; (B) mRNA levels of iNOS2 and Arg I; (C) protein levels of NF- $\kappa B$ (nuclear and plasma). ${ }^{* * *} \mathrm{P}<0.001$ ( $v s$. vehicle); ${ }^{* \#} \mathrm{P}<0.01$, \#\#苂 $<0.001$ ( $v s$. LPS + vehicle).

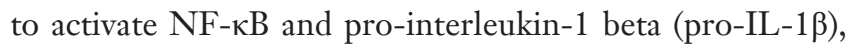
leading to neuroinflammation and age-related neurological condition (40), and LR4 has been shown to activate NF- $\mathrm{KB}$ and increase TNF- $\alpha$ and IL-6 (41). Huang et al. reported that TLR4 activated NF- $\kappa \mathrm{B}$ to induce IL-6/IL-8, leading to colon cancer progression (42). NF- $\kappa \mathrm{B}$ was activated in hepatocytes (43) and hepatic macrophages (44) during cholestatic injury. Downregulation of NF- $\mathrm{\kappa B}$ signaling is involved in the protective effects of chlorogenic acid innaphthylisothiocyanate (ANIT)-induced cholestasis and liver injury (45). Our results showed that silencing of TGR5 significantly increased TLR4 and promoted NF- $\mathrm{\kappa B}$ nuclear translocation, leading to the elevation of TNF- $\alpha$ and IL-6. In contrast, TGR 5 activation remarkably inhibited secretion of pro-inflammatory cytokines. These findings reveal a new role for TGR5 in CLDs, showing that its activation inhibits NF- $\kappa \mathrm{B}$ signaling to suppress inflammation in Kupffer cells. However, role of TGR5 in Kupffer cells and CLD, is controversial $(10,46-49)$. It has been reported the functions of TGR5 in other liver cells. It may regulate liver microcirculation in sinusoidal endothelial cells (SEC) (50), TGR5 mediates secretory, proliferative and antiapoptotic effects in cholangiocytes (51). Keitel et al. has suggested that TGR5 may regulate BA transport in gallbladder epithelial cells (52). Animal studies have supported the protective roles of TGR5 in various liver diseases. RDX8940, a TGR5 agonist improves liver steatosis and insulin sensitivity in a mouse model (5). TGR5 regulates type I and II Natural killer T (NKT) cell polarization and inhibits inflammation in a mouse model of hepatitis (6). TGR5 alleviated inflammation response and liver damage in a hepatic ischaemia-reperfusion injury model (7). Further 
studies exploring the possible function of TGR5 in the communication between Kupffer cells and other liver cells, such as cholangiocytes, gallbladder epithelial cells and hepatic stellate cells, during the pathogenesis of CLD will provide more relevant data.

Taken together, our results indicate that TGR5 activation protects against BDL-induced CLD by suppressing inflammation through inhibiting NF- $\mathrm{KB}$-dependent proinflammatory cytokine production and reducing ROS through activating $\mathrm{Nrf} 2 / \mathrm{HO}-1$ signaling. These findings demonstrate the importance of TGR5 in CLD and provide new insight into therapeutic strategies for CLD.

\section{Acknowledgments}

Funding: This study was supported by the National Natural Science Foundation of China (Youth Project) [81700537].

\section{Footnote}

Reporting Checklist: The authors have completed the ARRIVE reporting checklist. Available at https://dx.doi. org/10.21037/atm-21-2631

Data Sharing Statement: Available at https://dx.doi. org/10.21037/atm-21-2631

Conflicts of Interest: All authors have completed the ICMJE uniform disclosure form (available at https://dx.doi. org/10.21037/atm-21-2631). The authors have no conflicts of interest to declare.

Etbical Statement: The authors are accountable for all aspects of the work in ensuring that questions related to the accuracy or integrity of any part of the work are appropriately investigated and resolved. All procedures performed in this study involving human participants were in accordance with the Declaration of Helsinki (as revised in 2013). The Ethics Committee of The Affiliated Changzhou No. 2 People's Hospital of Nanjing Medical University approved all collections \{No. [2020] KY125-01\}. All patients provided written informed consent. All animal experiments were performed in accordance with the guidelines for animal care and use under the protocols approved by the Institutional Animal Care and Use Committee of The Affiliated Changzhou No. 2 People's Hospital of Nanjing Medical University.

Open Access Statement: This is an Open Access article distributed in accordance with the Creative Commons Attribution-NonCommercial-NoDerivs 4.0 International License (CC BY-NC-ND 4.0), which permits the noncommercial replication and distribution of the article with the strict proviso that no changes or edits are made and the original work is properly cited (including links to both the formal publication through the relevant DOI and the license). See: https://creativecommons.org/licenses/by-nc-nd/4.0/.

\section{References}

1. Trauner M, Meier PJ, Boyer JL. Molecular pathogenesis of cholestasis. N Engl J Med 1998;339:1217-27.

2. Marucci L, Baroni GS, Mancini R, et al. Cell proliferation following extrahepatic biliary obstruction. Evaluation by immunohistochemical methods. J Hepatol 1993;17:163-9.

3. Malik A, Kardashian AA, Zakharia K, et al. Preventative care in cholestatic liver disease: Pearls for the specialist and subspecialist. Liver Res 2019;3:118-27.

4. Liu C, Gao J, Liu J, et al. Intrahepatic cholestasis of pregnancy is associated with an increased risk of gestational diabetes and preeclampsia. Ann Transl Med 2020;8:1574.

5. Tag CG, Sauer-Lehnen S, Weiskirchen S, et al. Bile duct ligation in mice: induction of inflammatory liver injury and fibrosis by obstructive cholestasis. J Vis Exp 2015;(96):52438.

6. Bull LN, Vargas J. Serum bile acids in intrahepatic cholestasis of pregnancy: not just a diagnostic test. Hepatology 2014;59:1220-2.

7. Kim MJ, Suh DJ. Profiles of serum bile acids in liver diseases. Korean J Intern Med 1986;1:37-42.

8. Beuers U, Kremer AE, Bolier R, et al. Pruritus in cholestasis: facts and fiction. Hepatology 2014;60:399-407.

9. Kawamata Y, Fujii R, Hosoya M, et al. A G proteincoupled receptor responsive to bile acids. J Biol Chem 2003;278:9435-40.

10. Osawa Y, Seki E, Adachi M, et al. Role of acid sphingomyelinase of Kupffer cells in cholestatic liver injury in mice. Hepatology 2010;51:237-45.

11. Lv Q, Gao R, Peng C, et al. Bisphenol A promotes hepatic lipid deposition involving Kupffer cells M1 polarization in male mice. J Endocrinol 2017;234:143-54.

12. Liu CP, Zhang X, Tan QL, et al. NF- $\kappa$ B pathways are involved in M1 polarization of RAW 264.7 macrophage by polyporus polysaccharide in the tumor microenvironment. PLoS One 2017;12:e0188317.

13. Liu T, Zhang L, Joo D, et al. NF- $\kappa$ B signaling in inflammation. Signal Transduct Target Ther 2017;2:17023. 
14. Keitel V, Donner M, Winandy S, et al. Expression and function of the bile acid receptor TGR5 in Kupffer cells. Biochem Biophys Res Commun 2008;372:78-84.

15. Yang H, Zhou H, Zhuang L, et al. Plasma membranebound $\mathrm{G}$ protein-coupled bile acid receptor attenuates liver ischemia/reperfusion injury via the inhibition of toll-like receptor 4 signaling in mice. Liver Transpl 2017;23:63-74.

16. Pols TW, Nomura M, Harach T, et al. TGR5 activation inhibits atherosclerosis by reducing macrophage inflammation and lipid loading. Cell Metab 2011;14:747-57.

17. Wang YD, Chen WD, Yu D, et al. The G-protein-coupled bile acid receptor, Gpbar1 (TGR5), negatively regulates hepatic inflammatory response through antagonizing nuclear factor $\kappa$ light-chain enhancer of activated $B$ cells (NF-кB) in mice. Hepatology 2011;54:1421-32.

18. Copple BL, Jaeschke H, Klaassen CD. Oxidative stress and the pathogenesis of cholestasis. Semin Liver Dis 2010;30:195-204.

19. Booth DM, Murphy JA, Mukherjee R, et al. Reactive oxygen species induced by bile acid induce apoptosis and protect against necrosis in pancreatic acinar cells. Gastroenterology 2011;140:2116-25.

20. Fang Y, Han SI, Mitchell C, et al. Bile acids induce mitochondrial ROS, which promote activation of receptor tyrosine kinases and signaling pathways in rat hepatocytes. Hepatology 2004;40:961-71.

21. Deng L, Chen X, Zhong Y, et al. Activation of TGR5 Partially Alleviates High Glucose-Induced Cardiomyocyte Injury by Inhibition of Inflammatory Responses and Oxidative Stress. Oxid Med Cell Longev 2019;2019:6372786.

22. Guo J, Zheng L, Chen L, et al. Lipopolysaccharide

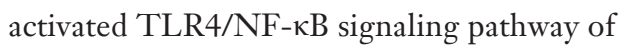
fibroblasts from uterine fibroids. Int J Clin Exp Pathol 2015;8:10014-25.

23. Lefebvre P, Cariou B, Lien F, et al. Role of bile acids and bile acid receptors in metabolic regulation. Physiol Rev 2009;89:147-91.

24. Jourdainne V, Péan N, Doignon I, et al. The Bile Acid Receptor TGR5 and Liver Regeneration. Dig Dis 2015;33:319-26.

25. Reich M, Deutschmann K, Sommerfeld A, et al. TGR5 is essential for bile acid-dependent cholangiocyte proliferation in vivo and in vitro. Gut 2016;65:487-501.

26. Keitel V, Görg B, Bidmon HJ, et al. The bile acid receptor TGR5 (Gpbar-1) acts as a neurosteroid receptor in brain.
Glia 2010;58:1794-805.

27. Wang XX, Edelstein MH, Gafter U, et al. G ProteinCoupled Bile Acid Receptor TGR5 Activation Inhibits Kidney Disease in Obesity and Diabetes. J Am Soc Nephrol 2016;27:1362-78.

28. Baird L, Dinkova-Kostova AT. The cytoprotective role of the Keap1-Nrf2 pathway. Arch Toxicol 2011;85:241-72.

29. Loboda A, Rojczyk-Golebiewska E, Bednarczyk-Cwynar $\mathrm{B}$, et al. Targeting nrf2-mediated gene transcription by triterpenoids and their derivatives. Biomol Ther (Seoul) 2012;20:499-505.

30. Romanoski CE, Che N, Yin F, et al. Network for activation of human endothelial cells by oxidized phospholipids: a critical role of heme oxygenase 1. Circ Res 2011;109:e27-41.

31. Chakraborty S, Balan M, Flynn E, et al. Activation of c-Met in cancer cells mediates growth-promoting signals against oxidative stress through $\mathrm{Nrf2}-\mathrm{HO}-1$. Oncogenesis 2019;8:7.

32. Li H, Yao W, Irwin MG, et al. Adiponectin ameliorates hyperglycemia-induced cardiac hypertrophy and dysfunction by concomitantly activating Nrf2 and Brg1. Free Radic Biol Med 2015;84:311-21.

33. Coussens LM, Werb Z. Inflammation and cancer. Nature 2002;420:860-7.

34. Tsalamandris S, Antonopoulos AS, Oikonomou E, et al. The Role of Inflammation in Diabetes: Current Concepts and Future Perspectives. Eur Cardiol 2019;14:50-9.

35. Lopez-Candales A, Hernández Burgos PM, HernandezSuarez DF, et al. Linking Chronic Inflammation with Cardiovascular Disease: From Normal Aging to the Metabolic Syndrome. J Nat Sci 2017;3:e341.

36. Hua F, Ma J, Ha T, et al. Activation of Toll-like receptor 4 signaling contributes to hippocampal neuronal death following global cerebral ischemia/reperfusion. J Neuroimmunol 2007;190:101-11.

37. Frank S, Copanaki E, Burbach GJ, et al. Differential regulation of toll-like receptor mRNAs in amyloid plaqueassociated brain tissue of aged APP23 transgenic mice. Neurosci Lett 2009;453:41-4.

38. Casula M, Iyer AM, Spliet WG, et al. Toll-like receptor signaling in amyotrophic lateral sclerosis spinal cord tissue. Neuroscience 2011;179:233-43.

39. Kawai T, Akira S. Signaling to NF-kappaB by Toll-like receptors. Trends Mol Med 2007;13:460-9.

40. Azam S, Jakaria M, Kim IS, et al. Regulation of Toll-Like Receptor (TLR) Signaling Pathway by Polyphenols in the Treatment of Age-Linked Neurodegenerative Diseases: Focus on TLR4 Signaling. Front Immunol 2019;10:1000. 
41. Teng W, Wang L, Xue W, et al. Activation of TLR4mediated NFkappaB signaling in hemorrhagic brain in rats. Mediators Inflamm 2009;2009:473276.

42. Huang HY, Zhang ZJ, Cao CB, et al. The TLR4/NF-кB signaling pathway mediates the growth of colon cancer. Eur Rev Med Pharmacol Sci 2014;18:3834-43.

43. Miyoshi H, Rust C, Guicciardi ME, et al. NF-kappaB is activated in cholestasis and functions to reduce liver injury. Am J Pathol 2001;158:967-75.

44. Fox ES, Kim JC, Tracy TF. NF-kappaB activation and modulation in hepatic macrophages during cholestatic injury. J Surg Res 1997;72:129-34.

45. Tan Z, Luo M, Yang J, et al. Chlorogenic acid inhibits cholestatic liver injury induced by $\alpha$-naphthylisothiocyanate: involvement of STAT3

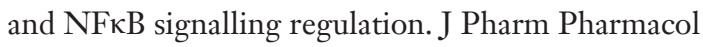
2016;68:1203-13.

46. Gehring S, Dickson EM, San Martin ME, et al. Kupffer cells abrogate cholestatic liver injury in mice. Gastroenterology 2006;130:810-22.

47. Abrahám S, Szabó A, Kaszaki J, et al. Kupffer cell blockade improves the endotoxin-induced microcirculatory inflammatory response in obstructive jaundice. Shock 2008;30:69-74.

48. Zandieh A, Payabvash S, Pasalar P, et al. Gadolinium chloride, a Kupffer cell inhibitor, attenuates hepatic injury in a rat model of chronic cholestasis. Hum Exp Toxicol 2011;30:1804-10.

49. Jones C, Badger SA, Hoper M, et al. Hepatic cytokine response can be modulated using the Kupffer cell blocker gadolinium chloride in obstructive jaundice. Int J Surg 2013;11:46-51.

50. Keitel V, Reinehr R, Gatsios P, et al. The G-protein coupled bile salt receptor TGR5 is expressed in liver sinusoidal endothelial cells. Hepatology 2007;45:695-704.

51. Keitel V, Häussinger D. TGR5 in cholangiocytes. Curr Opin Gastroenterol 2013;29:299-304.

52. Keitel V, Cupisti K, Ullmer C, et al. The membrane-bound bile acid receptor TGR5 is localized in the epithelium of human gallbladders. Hepatology 2009;50:861-70.

(English Language Editor: B. Draper)
Cite this article as: Yang H, Luo F, Wei Y, Jiao Y, Qian J, Chen S, Gong Y, Tang L. TGR5 protects against cholestatic

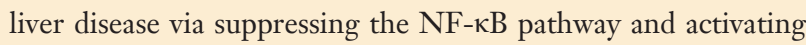
the Nrf2/HO-1 pathway. Ann Transl Med 2021;9(14):1158. doi: 10.21037/atm-21-2631 

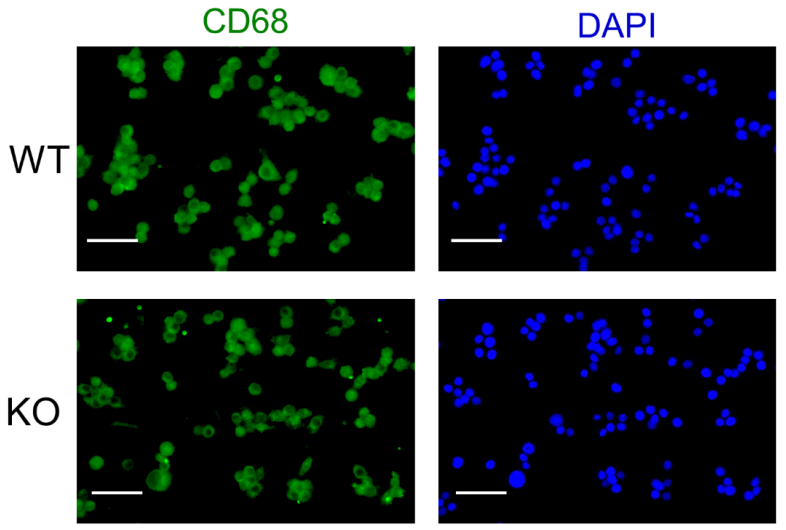

Figure S1 Characterization of Kupffer cells. Kupffer cells were isolated and CD68 immunofluorescent staining was used to analyze their purity (scale bar: $50 \mu \mathrm{m}$ ).

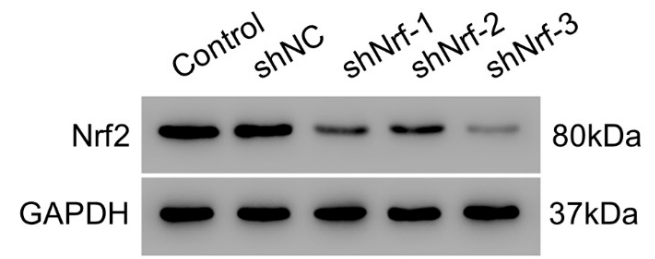

Figure S2 Nrf2 interference in primary Kupffer cells. shRNA targeting Nrf2 (shNrf2) or negative control shRNA (shNC) was used to silence Nrf2 in primary mouse Kupffer cells. Western blots were used to confirm the silencing efficiency.

Table S1 Target sequences of mouse Nrf2 shRNAs

\begin{tabular}{ll}
\hline shRNA & Target sequence \\
\hline shNrf2-1 & 5' GCAGGACATGGATTTGATT 3' \\
shNrf2-2 & 5' GCAAGACTTGGGCCACTTA 3' \\
shNrf2-3 & 5' CCTTGTATCTTGAAGTCTT 3' \\
\hline
\end{tabular}

Table S2 Primer sequences for real-time PCR

\begin{tabular}{lll}
\hline Gene & Forward primer & Reverse primer \\
\hline TGR5 & 5' GGCAAGCCTCATCATCACC 3' & 5' AAGTTGGGAGCCAAGTAGACG 3' \\
iNOS2 & 5' GAGCGAGTTGTGGATTGTC 3' & 5' CCAGGAAGTAGGTGAGGG 3' \\
Arg1 & 5' GCTTGCGAGACGTAGACC 3' & 5' CATCACCTTGCCAATCCC 3' \\
GAPDH & 5' GGATTGTCTGGCAGTAGCC 3' & 5' ATTGTGAAAGGCAGGGAG 3' \\
\hline
\end{tabular}

Table S3 Antibody list

\begin{tabular}{lcc}
\hline Primary antibody & Company & Catalog No. \\
\hline TGR5 & Abcam & Ab72608 \\
TLR4 & Abcam & Ab13556 \\
HO-1 & Abcam & ab13243 \\
NF- $\kappa$ B & Abcam & Ab16502 \\
Nrf2 & Abcam & Ab92946 \\
H3 & Abcam & Ab1791 \\
GAPDH & Cell Signaling Technology & $\# 5174$ \\
\hline
\end{tabular}

